\title{
Chemotherapy in the Covid-19 era: the patient's perception
}

\author{
Maeve A. Hennessy ${ }^{1,2} \cdot$ Anna Linehan ${ }^{1,2} \cdot$ Darren Cowzer $^{1,2} \cdot$ Zachary L. Coyne $^{1,2} \cdot$ Orla Fitzpatrick $^{1} \cdot$ Fiona Barrett $^{1}$. \\ Roisin Ni Dhonaill ${ }^{1} \cdot$ Bryan T. Hennessy ${ }^{1,2} \cdot$ Liam Grogan $^{1,2} \cdot$ Patrick G. Morris $^{1,2} \cdot$ Oscar S. Breathnach $^{1,2}$
}

Received: 11 October 2020 / Accepted: 3 December 2020 / Published online: 15 January 2021

(C) Royal Academy of Medicine in Ireland 2021

\begin{abstract}
Background The Covid-19 pandemic poses significant challenges for the management of patients with cancer. In our institution, we adapted our delivery of outpatient systemic anti-cancer therapy (SACT) by introducing a number of 'risk-reducing' measures including pre-assessment screening.

Aims We sought to evaluate the experience and perceptions of patients with cancer undergoing SACT during the Covid-19 pandemic.

Methods Patients on SACT during the Covid-19 pandemic were eligible for participation. Data were collected by anonymous survey over a 1 week period during the most intensive phase of government restrictions. Patients were asked questions under three headings: perceived risk of infection exposure, changes to treatment plan and psychological impact of Covid-19.

Results One hundred patients were assessed, $60 \%$ were male, $41 \%$ were $>65$ years of age and $67 \%$ had advanced cancer. Eleven percent of patients were living alone. Fifty-seven percent reported feeling at increased risk in general of contracting Covid-19. Sixtyeight percent of patients did not feel worried about contracting Covid-19 in the hospital. Ninety-two percent of patients reported wanting to continue on SACT as originally planned. Fifty-eighty percent felt isolated and $40 \%$ reported increased anxiety.

Conclusion Though patients on active treatment for cancer during the Covid-19 pandemic reported increased anxiety and feelings of isolation due to Covid-19, the majority of patients wanted to continue SACT as originally planned. Patients would benefit from enhanced psycho-oncological supports in the event of a prolonged Covid-19 pandemic.
\end{abstract}

Keywords Cancer $\cdot$ Chemotherapy $\cdot$ Covid-19 pandemic $\cdot$ Psychosocial impact

\section{Introduction}

Coronavirus disease 2019 (Covid-19) has resulted in a worldwide pandemic and has led to the rapid transformation of healthcare services around the globe. The symptoms of the virus range from asymptomatic cases to mild or moderate infection resembling the common cold or influenza. In some instances, however, Covid-19 causes significant illness such as severe acute respiratory distress or multi-organ dysfunction, resulting in admission to intensive care, and infection may be fatal in a subset of patients [1]. Those with underlying health conditions or those who are immunocompromised are

Oscar S. Breathnach

osbreathnach@beaumont.ie

1 Medical Oncology Department, Beaumont Hospital, Dublin, Ireland

2 Cancer Clinical Trials and Research Unit, Beaumont Hospital, Dublin, Ireland particularly at risk for adverse outcomes following infection with Covid-19 [2].

Consequently, the pandemic poses significant challenges for the management of patients with cancer, and in particular, those undergoing systemic anti-cancer therapy (SACT). This is a group who are at increased risk of infection with Covid-19 for several reasons, including the underlying disease process itself, the immunosuppressive nature of SACT, as well as the associated number of visits to hospital while undergoing treatment. Published reports from the initial outbreak in China found that patients with cancer who were infected with Covid-19 had a higher risk of death compared to patients without cancer [3, 4].

A variety of mental health disorders such as anxiety, depression and panic disorder due to the Covid-19 pandemic have been observed in the general population $[5,6]$. Patients with cancer have the additional psychological challenges of dealing with a cancer diagnosis during a pandemic, and this is a cohort that has previously been shown to have increased rates of anxiety and depression as compared to the general population $[7,8]$. 
Against this background, our institution adapted our delivery of outpatient SACT by introducing a number of 'riskreducing' measures including pre-assessment screening. We sought to evaluate our patients' experiences while on SACT during the Covid-19 pandemic and to gain an insight into their perception of the impact the pandemic has had on their oncological care and psychological wellbeing.

\section{Methods}

Patients on active SACT attending the oncology day ward for treatment during the Covid-19 pandemic were eligible for participation. Patients attending the day ward for the following reasons were excluded: (1) acutely unwell reviews, (2) 'new patient' visits where patients were attending for chemotherapy education and informed consent and (3) attendances for day case procedures such as biopsies and interventional radiology procedures. Multiple visits by the same patient, e.g. for chemotherapy protocols spanning more than 1 day such as testicular cancer regimens, were taken into consideration and such patients were only included once. Data collection took place from 11 May 2020 to 18 May 2020, during the most intensive phase of government restrictions.

Data were collected by anonymous survey prior to receipt of treatment. Socio-demographics and clinical characteristics were recorded. These included age, sex, type of cancer, cancer stage and type of cancer treatment. Information on social supports and living arrangements, compliance with government guidelines on self-isolation, access to community health services and engagement with news and social media was also collected.

In order to assess how patients perceived their care during Covid-19, they were asked to rate their agreement with statements under three general headings: changes to treatment plan, perceived risk of infection exposure and psychological impact of Covid-19.

\section{Changes to treatment plan}

Patients were asked if their oncologist had made any changes to their treatment plan due to Covid-19, if they themselves wanted to make any changes to their treatment plan because of Covid-19 and if they themselves had delayed or avoided seeking medical attention (e.g. presentation to emergency department) due to Covid-19. Answers were given as yes, no or unsure.

\section{Risk of infection exposure}

Patients were asked if they worried about being at increased risk of infection with Covid-19, if they worried about contracting Covid-19 in a healthcare facility and if they worried about contracting Covid-19 in the community. Answers were based on a 5-point Likert scale ranging from 1 (strongly disagree) to 5 (strongly agree).

\section{Psychological impact}

Patients were asked if Covid-19 had affected their level of anxiety, depression, uncertainty regarding the future and feelings of isolation, as compared to usual. Answers were based on a 5-point Likert scale as above.

\section{Results}

There were 207 scheduled day ward attendances during the time period studied. Of these, 21 patients did not attend (DNA), 20 were repeat visits, 18 were new patient appointments, 6 were acutely unwell reviews and 4 were attending for day case procedures. This left a possible sample of $138 \mathrm{pa}-$ tients and of these, 100 patients on SACT who attended the oncology day ward for treatment participated. Sixty percent were male and $41 \%$ were over 65 years of age. Just over two thirds of participants, $67 \%$, had advanced, incurable cancer. Fifty-nine percent of patients were receiving SACT in the form of chemotherapy. The most common type of cancer was colorectal (25\%), followed by breast $(17 \%)$ and lung (14\%) (Table 1).

In terms of social supports, the majority of patients were living with others, while $11 \%$ of patients reported living alone. Almost all patients, $95 \%$, reported that they had family/friends available to help with daily activities such as shopping and transport to medical appointments. Almost all patients, 95\%, reported practising social isolation, which was in keeping with government advice at the time of the study (Table 1).

Regarding changes to treatment plan, the vast majority of patients reported wanting to continue on their SACT as originally planned, $15 \%$ of patients reported that their oncologist had changed their SACT due to Covid-19 (Fig. 1). Ten percent of patients reported that due to the Covid-19 pandemic, they had opted to delay medical attention if unwell at home.

When asked about the risk of contracting Covid-19, over half of the patients agreed or strongly agreed that they felt at increased risk in general of falling ill with the virus. Nearly two thirds of patients either disagreed or strongly disagreed with feeling at increased risk of Covid-19 in hospital (Fig. 2).

Participants self-reported increased rates of anxiety and depression in comparison with before the pandemic. Forty percent of patients agreed or strongly agreed that they had increased levels of anxiety than before and $23 \%$ reported increased depression. Fifty-eight percent agreed or strongly agreed that they felt more isolated than they did before the pandemic (Fig. 3). 
Table 1 Baseline characteristics of the 100 oncology patients on SACT surveyed during the Covid-19 pandemic, between 11 May 2020 and 18 May 2020

\begin{tabular}{|c|c|}
\hline & $\begin{array}{l}\text { All patient } \\
N=100\end{array}$ \\
\hline \multicolumn{2}{|l|}{ Age } \\
\hline $18-30$ & - \\
\hline $31-64$ & 59 \\
\hline $65-80$ & 39 \\
\hline$>80$ & 2 \\
\hline \multicolumn{2}{|l|}{ Gender } \\
\hline Male & 60 \\
\hline Female & 40 \\
\hline \multicolumn{2}{|l|}{ Cancer type } \\
\hline Lung & 14 \\
\hline Breast & 17 \\
\hline Colorectal & 25 \\
\hline Upper GI & 9 \\
\hline Prostate & 9 \\
\hline $\mathrm{CNS}$ & 9 \\
\hline Other & 17 \\
\hline \multicolumn{2}{|l|}{ Stage } \\
\hline Early & 24 \\
\hline Advanced & 67 \\
\hline Unknown & 9 \\
\hline \multicolumn{2}{|l|}{ Treatment } \\
\hline Chemotherapy & 59 \\
\hline Immunotherapy & 4 \\
\hline Targeted Tx & 6 \\
\hline Hormonal Tx & 6 \\
\hline Unknown & 25 \\
\hline \multicolumn{2}{|l|}{ Social supports } \\
\hline Living alone & 11 \\
\hline Self-isolating & 95 \\
\hline Help with shopping/transport & 95 \\
\hline
\end{tabular}

\section{Discussion}

In this study, a large number of patients over 65 years of age were included, $41 \%$, and the majority of the population surveyed had advanced malignancy. In general, patients reported complying with government guidelines, reported good social supports and were keen to continue on their treatment as originally planned. Interestingly, the majority of patients were not concerned regarding increased risk of Covid-19 when attending the hospital for their outpatient SACT. The study took place following the adapted delivery of SACT and the implementation of 'risk-reducing' measures in our institution in March 2020.

Such measures included contacting all patients via telephone the day prior to oncology day ward attendance to screen for symptoms of cough, shortness of breath, fever, sore throat, new loss of taste or smell, or other flu-like symptoms. If patients were symptomatic, further triage was undertaken in order to determine if an appointment was necessary, if an urgent Covid-19 test needed to be arranged or if the patient could be managed from home. This was in line with the American Society of Clinical Oncology (ASCO) and the European Society of Medical Oncology (ESMO) guidance [9, 10]. We also ran an in-person, nurse-led triage on the day of treatment which included assessment of vital signs and temperature checks. We arranged a separate access to the day ward, away from the main hospital entrance, reserved for sole use of Oncology and Haematology patients. The waiting room was adjusted to facilitate social distancing and patients were given strict appointment times in order to minimise the number of people in attendance at all times. Visiting restrictions were also in place and patients were not allowed to have another person with them, other than in exceptional circumstances. Our findings appear to suggest that the safety modifications and procedures implemented pre-empted patients concerns regarding the risk of hospital acquired Covid-19 infection. In general, we found that the majority of patients were willing to attend hospital, continue treatment and reported a lack of concern for contracting Covid-19 in a healthcare environment.

The continued safe delivery of cancer therapies was a priority in our institution during the pandemic. In relation to planned SACT, we aimed to minimise patient's exposure to Covid-19 without compromising treatment efficacy. We based our decisions on current evidence that supported the adaptation of treatment regimens and we were guided by ASCO and ESMO publications on cancer care delivery during the Covid-19 pandemic $[9,10]$. Our patients' care plans were determined following review of current international clinical practice, and on an individual basis by the treating oncologist, taking factors such as disease status, treatment intent (curative versus palliative), age, frailty, comorbidities and patient preference into account [11].

The challenge of determining the optimal SACT regimen in the context of a global pandemic was discussed with patients, and we explained to all affected that the balance of benefit versus risk had shifted. We discussed that current evidence supports the adaptation of treatment regimens in many cases, and alternative strategies, such as oral drugs, shorter administration times and larger intervals between doses, were considered and offered to patients $[9,10,12,13]$. Where possible, immunotherapy was prescribed at extended dosage intervals, e.g. 6 weekly pembrolizumab rather than 3 weekly $[12,13]$. This followed the recent approval of a 6-week dosing schedule for pembrolizumab across all monotherapy indications, by the 
Fig. 1 Changes made to SACT treatment plan due to the Covid19 pandemic, as perceived by patients

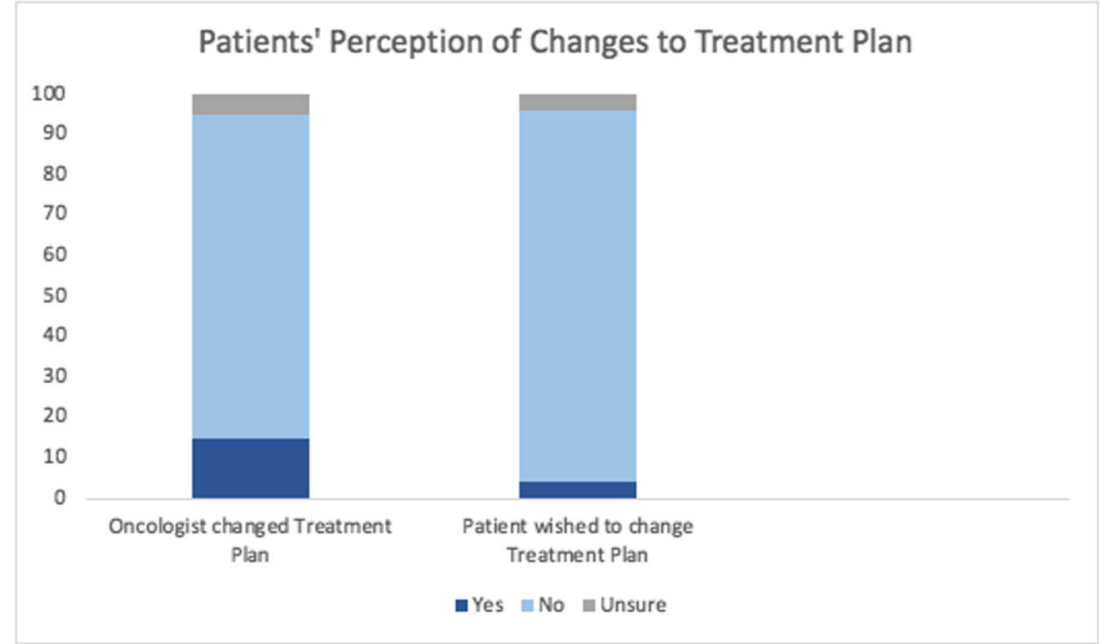

European Commission [14]. For patients with early stage breast cancer, we discussed the early discontinuation of adjuvant trastuzumab on the basis of a number of Phase III randomised trials which suggest that, in low-risk patients, it is reasonable to stop trastuzumab before the standard duration of 1 year [15, 16]. In patients with both early stage and advanced colorectal cancer, we considered switching from the 2 weekly FOLFOX regimen with 5-fluorouracil (5-FU) continuous infusion, to the 3 weekly CAPOX regimen, which is at least as effective, utilises oral capecitabine rather than infusional 5-FU and reduces the need for physical attendances to the hospital [17]. Some clinically stable patients on palliative maintenance therapies, such as bevacizumab in advanced colorectal or ovarian cancer, were offered treatment breaks [9, 10].

There has been a reluctance noted among the general population to seek medical attention when unwell and in many cases patients have stayed at home in order to avoid admission to hospital during the pandemic. This may be due to strong governmental messaging regarding the importance of selfisolating and limiting social contacts, coupled with media warnings regarding hospital over-crowding. Indeed, this is a possible limitation of our study, in that it is likely that those who felt most at risk were not included, as they may not have presented for their scheduled appointment or may not have been willing to engage with the healthcare system. Research published by the Economic and Social Research Institute (ESRI) Ireland in May 2020 demonstrated a sharp fall in emergency department (ED) attendances at the onset of the Covid19 outbreak in Ireland, with the average number of daily attendances at the end of March 2020 approximately half of what it was at the start of March [18]. This included a decline in the number of attendances classified as very urgent or immediate, suggesting that Covid-19 is having significant impacts on people's ability and willingness to access timely healthcare [18]. Despite this, $90 \%$ of patients in this study reported that they had not delayed seeking medical attention. Again, this may reflect some of the safety changes implemented in our institution.

In addition to the above challenges, the Covid-19 pandemic has brought worldwide mental health distress and a recent
Fig. 2 Perceived risk of exposure to Covid-19 in general and in the hospital, as reported by patients

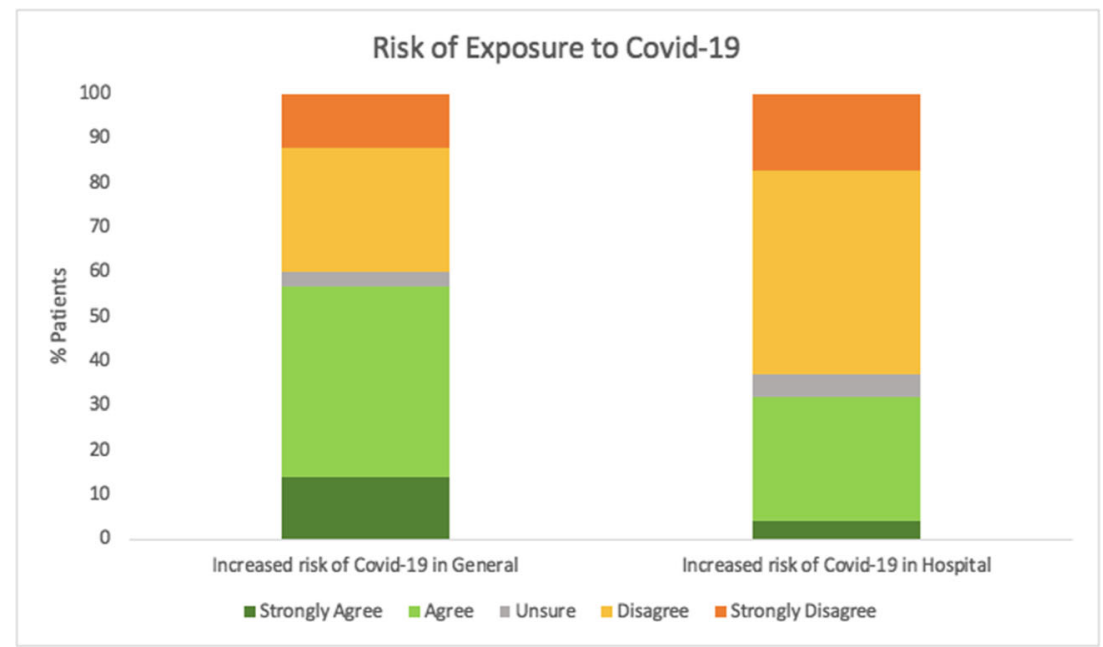


Fig. 3 Psychological Impact of Covid-19, as reported by patients

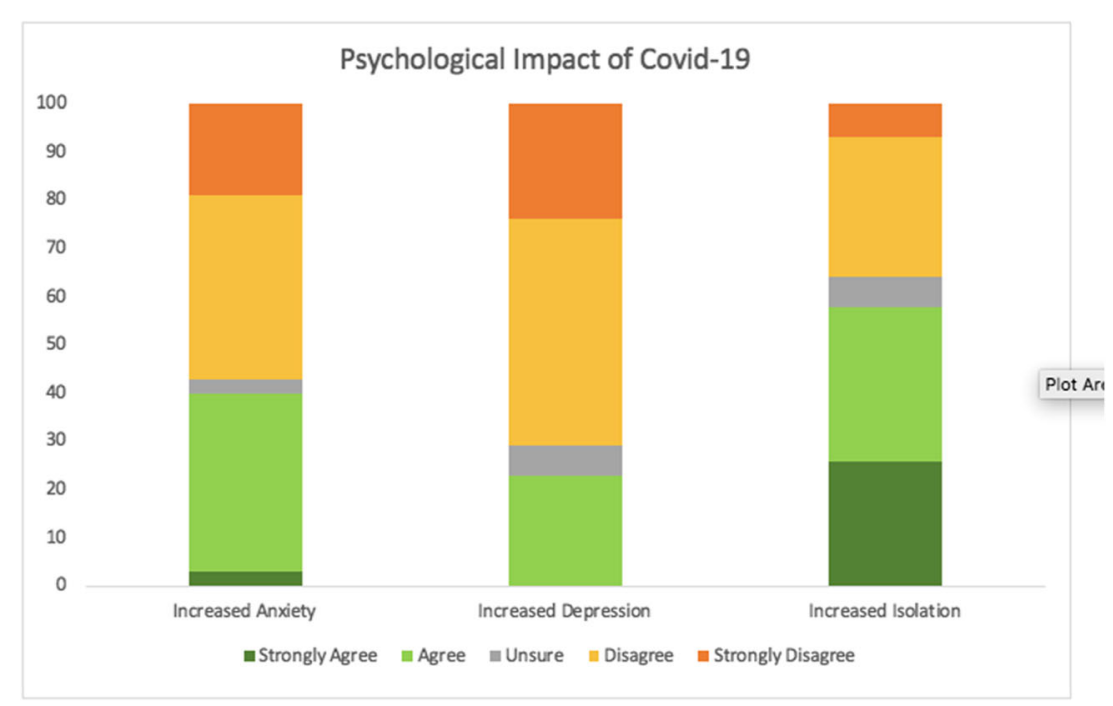

study from China reported increased suffering from issues such as stress, anxiety and depression [19]. Patients who are self-isolating are likely to have greater levels of anxiety, irritability and depression, especially when restrictions are implemented for prolonged periods [20]. The social isolation and being confined to the home can cause loneliness, which is associated with a higher risk of mortality in cancer patients [21]. The impact of Covid-19 on more vulnerable populations is not known and this was something we wished to address in our survey. Indeed $40 \%$ of participants in our study reported higher levels of anxiety than before the pandemic and 58\% reported feeling more isolated.

Fear that the pandemic could cause a delay in oncological treatments is a factor that has been noted in a number of surveys carried out in other countries. A survey of 86 women recently diagnosed with breast cancer was performed in an Italian University Hospital in March 2020 during the national lockdown. Seventy-one percent of participants reported concern that the pandemic could cause a delay in their oncological treatments and $56 \%$ were fearful that as cancer patients they could be more vulnerable to infection compared to the general population [22]. Results from a study in the Netherlands found that most patients were very concerned in terms of their oncological treatment or follow-up and $30 \%$ of patients reported that the pandemic had consequences for their oncological treatment or follow-up [23]. A survey to better understand the impact of Covid-19 on US cancer patients and survivors was set up by the American Cancer Society Cancer Action Network (ACS CAN) in March 2020. This found that $50 \%$ of cancer patients and survivors reported some impact to their health care due to the Covid-19 pandemic. Twenty-seven percent of patients who were on SACT reported treatment delays, and $13 \%$ of those on SACT had care delayed without knowledge of when it will be rescheduled [24]. The 'Covid-19 Pandemic Impact on Patients With Cancer-a Danish
Survey (COPICADS)' is an observational study with an estimated enrolment of 5000 participants and a target follow-up duration of 12 months. The study aims to investigate the overall quality of life for patients with cancer during the Covid-19 pandemic, specifically focusing on the emotional impact, and we await the results with interest [25].

\section{Conclusion}

The Covid-19 pandemic has added an increased complexity to the delivery of cancer care and the long-term effects on the survival and quality of life of patients with cancer are still not known. Oncology patients warrant particular attention as they are vulnerable to severe outcomes of Covid-19 infection, due to both their underlying malignancy and various anti-cancer treatments. We found that although patients on active treatment for cancer during the Covid-19 pandemic reported increased anxiety and feelings of isolation due to Covid-19, the majority of patients wanted to continue on their SACT as originally planned. Our finding would suggest that patients would benefit from enhanced psycho-oncological supports in the event of a prolonged Covid-19 pandemic.

\section{Compliance with ethical standards}

Conflict of interest The authors declare that they have no conflicts of interest.

\section{References}

1. World Health Organisation (2020) Coronavirus disease (COVID2019) situation reports, 2020. https://www.who.int/emergencies/ diseases/novel-coronavirus-2019/situation-reports. Accessed 11 September 2020 
2. Zhou F, Yu T, Du R et al (2020) Clinical course and risk factors for mortality of adult inpatients with COVID-19 in Wuhan, China: a retrospective cohort study. Lancet Oncol 395:1054-1062

3. Liang W, Guan W, Chen R et al (2020) Cancer patients in SARSCoV-2 infection: a nationwide analysis in China. Lancet Oncol 2020 21(3):335-337

4. Yu J, Ouyang W, Chua MLK, et al (2020) SARS-CoV-2 transmission in patients with cancer at a tertiary care hospital in Wuhan, China. JAMA Oncol 2020 6(7):1108-1110. https://doi.org/10. 1001/jamaoncol.2020.0980

5. Holmes EA, O'Connor RC, Perry VH et al (2020) Multidisciplinary research priorities for the COVID-19 pandemic: a call for action for mental health science. Lancet Psychiatry 2020 7:547-560. https://doi.org/10.1016/S2215-0366(20)30168-1

6. Galea S, Merchant RM, Lurie N (2020) The mental health consequences of COVID-19 and physical distancing. JAMA Intern Med 180:817-818. https://doi.org/10.1001/jamainternmed.2020.1562

7. Akechi T, Kugaya A, Okamura H et al (1999 Oct) (1999) Suicidal thoughts in cancer patients: clinical experience in psycho-oncology. Psychiatry Clin Neurosci 53(5):569-573. https://doi.org/10.1046/j. 1440-1819.1999.00607.x

8. Ng HS, Roder D, Koczwara B et al (2018) Comorbidity, physical and mental health among cancer patients and survivors: an Australian population-based study. Asia-Pac J Clin Oncol 2018(14):e181-e192. https://doi.org/10.1111/ajco.12677

9. American Society of Clinical Oncology (2020) ASCO special report: a guide to cancer care delivery during the Covid-19 pandemic. https:// www.asco.org/sites/new-www.asco.org/files/content-files/2020ASCO-Guide-Cancer-COVID19.pdf Accessed 11 September 2020

10. Curigliano G, Banerjee S, Cervantes A et al (2020) Managing cancer patients during the COVID-19 pandemic: an ESMO Interdisciplinary Expert Consensus. Ann Oncol 31(10):13201335. https://doi.org/10.1016/j.annonc.2020.07.010

11. Hanna TP, Evans GA, Booth CM (2020) (2020) Cancer, COVID19 and the precautionary principle: prioritizing treatment during a global pandemic. Nat Rev Clin Oncol 17(5):268-270. https://doi. org/10.1038/s41571-020-0362-6

12. Waisberg F, Enrico D, Angel M et al (2020) Cancer treatment adaptations in the COVID-19 era. JCO Oncology Practice 16, no. 6 (June 01, 2020) 305-307. https://doi.org/10.1200/OP.20.00218

13. Jazieh AR, Chan SL, Curigliano G et al (2020) Delivering cancer care during the COVID-19 pandemic: recommendations and lessons learned from ASCO global webinars. JCO Global Oncol 2020(6):1461-1471

14. Merck. European Medicines Agency adopts positive opinion for Merck's KEYTRUDA (pembrolizumab) for six-week dosing schedule across all current monotherapy indications. https:// investors.merck.com/news/press-release-details/2019/European-
Medicines-Agency-Adopts-Positive-Opinion-for-MercksKEYTRUDA-pembrolizumab-for-Six-Week-Dosing-ScheduleAcross-All-Current-Monotherapy-Indications/default.aspx

15. Conte P, Frassoldati A, Bisagni G et al (2018) Nine weeks versus 1 year adjuvant trastuzumab in combination with chemotherapy: final results of the phase III randomized Short-HER study. Ann Oncol 29:2328-2333 Medline, Google Scholar

16. Earl HM, Hiller L, Vallier A-L et al (2019) 6 versus 12 months of adjuvant trastuzumab for HER2-positive early breast cancer (PERSEPHONE): 4-year disease-free survival results of a randomised phase 3 non-inferiority trial. Lancet 393:2599-2612

17. Guo Y, Xiong B-H, Zhang T, Cheng Y et al (2016) XELOX vs. FOLFOX in metastatic colorectal cancer: an updated meta-analysis. Cancer Investig 34:94-104

18. Brick A, Walsh B, Keegan C et al (2020) ESRI special article: COVID-19 and emergency department attendances in Irish public hospitals. https://doi.org/10.26504/qec2020may_SA lyons Accessed 12 September 2020

19. Ahmed MZ, Ahemd O, Aibao Z et al (2020) Epidemic of COVID19 in China and associated psychological problems. Asian J Psychiatr. 2020 51:102092. https://doi.org/10.1016/j.ajp.2020. 102092

20. Brooks SK, Webster RK, Smith LE et al (2020) The psychological impact of quarantine and how to reduce it: rapid review of the evidence. Lancet Oncol. 2020 Mar 14 395(10227):912-920

21. D'Ippolito S, Ambrosini E, Shams M et al (2017) The effect of loneliness on cancer mortality. Ann Oncol 2017(28):vi82. https:// doi.org/10.1093/annonc/mdx434

22. Magno S, Linardos M, Carnevale S et al (2020) The impact of the COVID-19 pandemic on breast cancer patients awaiting surgery: observational survey in an Italian University hospital. Breast J 26: 1597-1602. https://doi.org/10.1111/tbj.13889

23. de Joode K, Dumoulin DW, Engelen V et al (2020) Impact of the COVID-19 pandemic on cancer treatment: the patients' perspective. European Journal of Cancer; Published online 4 July 2020. https://doi.org/10.1016/j.ejca.2020.06.019

24. The ASCO Post. Survey shows COVID-19 pandemic is affecting patients' access to cancer care. Available from: https://ascopost. com/news/april-2020/survey-shows-covid-19-pandemic-isaffecting-patients-access-to-cancer-care/. Accessed 09 Nov 2020

25. NIH ClinicalTrials.Gov. COVID-19 pandemic impact on patients with cancer - a Danish survey (COPICADS). Available from: https://clinicaltrials.gov/ct2/show/NCT04389996 . Accessed 09 Nov 2020

Publisher's note Springer Nature remains neutral with regard to jurisdictional claims in published maps and institutional affiliations. 